\title{
The impact of extreme obesity on outcomes after left ventricular assist device implantation
}

\author{
Gardner Yost, Laura Coyle, Colleen Gallagher, Nicole Graney, Roxanne Siemeck, Antone Tatooles, \\ Patroklos Pappas, Geetha Bhat
}

Advocate Christ Medical Center, Heart Institute, Heart and Vascular Institute Administration, Oak Lawn, IL 60453, USA

Contributions: (I) Conception and design: G Yost, L Coyle, G Bhat; (II) Administrative support: A Tatooles, P Pappas; (III) Provision of study materials or patients: L Coyle; (IV) Collection and assembly of data: C Gallagher, N Graney, R Siemeck, L Coyle; (V) Data analysis and interpretation: G Yost, L Coyle, G Bhat; (VI) Manuscript writing: All authors; (VII) Final approval of manuscript: All authors.

Correspondence to: Geetha Bhat, PhD, MD. 4440 W. $95^{\text {th }}$ Street, OPP $6^{\text {th }}$ Floor, Heart and Vascular Institute Administration, Oak Lawn, IL 60453 , USA. Email: Geetha.Bhat@advocatehealth.com.

\begin{abstract}
Background: The association between extreme body mass index (BMI) and outcomes in left ventricular assist device (LVAD) patients has not been well established. With the commercial use of LVADs a larger number of patients with a BMI $>40$ have undergone device implantation. The purpose of this study was to evaluate the short and long-term outcomes of LVAD patients with extreme obesity.

Methods: A retrospective review of all patients $(n=383)$ at our center who received a LVAD as primary implant between 2005-2015 was performed. Demographics, preoperative laboratory values, and postoperative outcomes were analyzed. Patients were divided into three groups based on BMI $\left(\mathrm{kg} / \mathrm{m}^{2}\right)$ classification (group 1: $\leq 25$; group 2: 25 to 35; group 3: $\geq 35$ ) and compared using one-way analysis of variance (ANOVA), Kruskal-Wallis and Chi-squared analysis as appropriate.

Results: Comparison of postoperative outcomes demonstrated an increased risk of respiratory failure and right ventricular (RV) failure in patients with a $\mathrm{BMI} \geq 35$ (range, 35-59). Length of stay, sternal infection, driveline/pocket infection, systemic infection, GI-bleeding, and neurological events within the first year of device therapy were not related to BMI. Survival at 30-day, 1- and 2-year was not significantly different among the three groups. The group with the smallest BMI demonstrated an increased risk for re-operative bleeding.

Conclusions: Despite an increased risk of early morbidity in patients with extreme obesity, long term survival was not significantly different between the BMI groups. Careful consideration is recommended when evaluating patients with an excessive BMI for LVAD therapy although it should not be a contraindication for device placement.
\end{abstract}

Keywords: Obesity; left ventricular assist device (LVAD); outcomes

Submitted Jun 13, 2017. Accepted for publication Jul 25, 2017.

doi: $10.21037 /$ jtd.2017.10.52

View this article at: http://dx.doi.org/10.21037/jtd.2017.10.52

\section{Introduction}

Obesity is a widely recognized risk factor for the development of numerous chronic diseases including hypertension, diabetes, and heart failure. As a result, the increasing prevalence of obesity, both in the USA and worldwide, poses both a significant public health concern and a challenging hurdle in the management of heart failure (1-3). At this time, the prevalence of obese patients with advanced heart failure who require surgical intervention with heart transplantation or left ventricular assist device (LVAD) therapy is increasing, generating the need for improved clinical protocols for the management of these patients (4). Despite well-established evidence that obesity is related to poor outcomes following 
heart transplantation, there remains a relative paucity of data concerning the effect of body mass index (BMI) on outcomes following $\operatorname{LVAD}(5,6)$. Several studies have indicated that obese patients may have increased rates of LVAD thrombosis and infections, but have survival rates similar to normal and underweight patients. These reports have generated varied results, and as a result, selection and management of LVAD patients with high BMIs remains challenging (7-9). At present there is only one other published study which investigated outcomes in patients with $\mathrm{BMI} \geq 35 \mathrm{~kg} / \mathrm{m}^{2}$ (10). The present study sought contribute to the current knowledge base regarding LVAD implantation in obese patients and to evaluate long term outcomes in patients with extreme obesity, defined as $\mathrm{BMI} \geq 35 \mathrm{~kg} / \mathrm{m}^{2}$.

\section{Methods}

This institutional review board approved study included 384 patients who underwent continuous flow LVAD implantation at our center between 2005 and 2015. All patients were implanted with either the HeartMate II or HeartWare HVAD. The patients were divided into 3 groups based on preoperative BMI. Group 1: underweight and healthy (BMI $<25 \mathrm{~kg} / \mathrm{m}^{2}$ ), group 2: pre-obesity and obesity $\left(25 \mathrm{~kg} / \mathrm{m}^{2} \leq \mathrm{BMI}<35 \mathrm{~kg} / \mathrm{m}^{2}\right)$, and group 3 : extreme obesity (BMI $\geq 35 \mathrm{~kg} / \mathrm{m}^{2}$ ).

\section{Variable selection}

Preoperative demographic data was collected for all patients and included age, gender, ethnicity, hypertension, diabetes mellitus (DM), atrial fibrillation, ventricular tachycardia, chronic obstructive pulmonary disease (COPD), obstructive sleep apnea (OSA), chronic kidney disease (CKD), and etiology of heart failure. Laboratory and hemodynamic data were collected prior to LVAD implantation and included: serum sodium, creatinine, total bilirubin, blood urea nitrogen (BUN), b-type natriuretic peptide (BNP), albumin, white cell count, hemoglobin, prealbumin, left ventricular ejection fraction (LVEF), central venous pressure (CVP), pulmonary capillary wedge pressure (PCWP), mean pulmonary artery pressure (MPAP), cardiac output (CO), and mean arterial pressure (MAP). Outcomes data including length of hospital stay and survival were collected for all patients. Adverse events included re-operative bleeding, right ventricular (RV) failure, respiratory failure, sternal infection, pocket or driveline infection, systemic infection, neurological events, and gastrointestinal bleeding. RV failure was defined as need for RV assist device or greater than 14 days of postoperative inotropic support.

\section{Statistical analysis}

Continuous variables were expressed as means and standard deviations when their distributions expressed normality and as medians and interquartile ranges when distributions were skewed. Demographics, frequencies of comorbidities, laboratory values, hemodynamics, ventricular dimensions, and LVEFs were compared between groups using 1-way analysis of variance (ANOVA) for normally distributed continuous variables. The Kruskal-Wallis test was used for non-parametric data. Categorical data were compared using Chi-square testing. Survival and length of stay were compared between groups using Kaplan-Meier analysis with non-parametric log-rank tests for significance. Multivariable Cox regression analysis was performed, adjusting for baseline characteristics that differed between the BMI groups by univariate analysis (age, DM, OSA, creatinine, BNP, albumin, CVP, MPAP, and CO). A $\mathrm{P}<0.05$ was considered statistically significant. Statistical operations were performed using SPSS version 20 (IBM, Chicago, IL, USA).

\section{Results}

\section{Baseline characteristics}

The patients included in this study had preoperative BMI ranging from 16.30 to $59.30 \mathrm{~kg} / \mathrm{m}^{2}$. The average BMI was $28.67 \pm 7.00 \mathrm{~kg} / \mathrm{m}^{2}$. Baseline demographics, comorbidities, laboratory values, and hemodynamics are provided in Table 1. Of the 383 patients included in this study, 118 (30.8\%) were underweight or normal weight (group 1), 196 (51.2\%) were pre-obese or obese (group 2), and 69 (18.0\%) were extremely obese (group 3). Patients in group 3 were significantly younger $(\mathrm{P}<0.001)$, had higher rates of DM $(\mathrm{P}=0.003)$, and OSA $(\mathrm{P}<0.001)$ than patients in groups 1 or 2 . Patients in group 3 also had higher CVP $(\mathrm{P}=0.001)$ and MPAP $(\mathrm{P}=0.048)$ than patients in groups 1 and 2. No significant differences in LVEF, or PCWP were noted. While creatinine levels were higher in groups 2 and $3(\mathrm{P}=0.001)$, group 3 had significantly better albumin $(\mathrm{P}=0.028)$ and $\mathrm{BNP}(\mathrm{P}<0.001)$ levels compared to groups 1 and 2 .

\section{Survival}

Survival was evaluated at 30 days, 1 year, 2 years, and 3 years after LVAD implantation. There were no differences 
Table 1 Preoperative demographic, laboratory, hemodynamic, and echocardiographic data compared for BMI groups 1-3

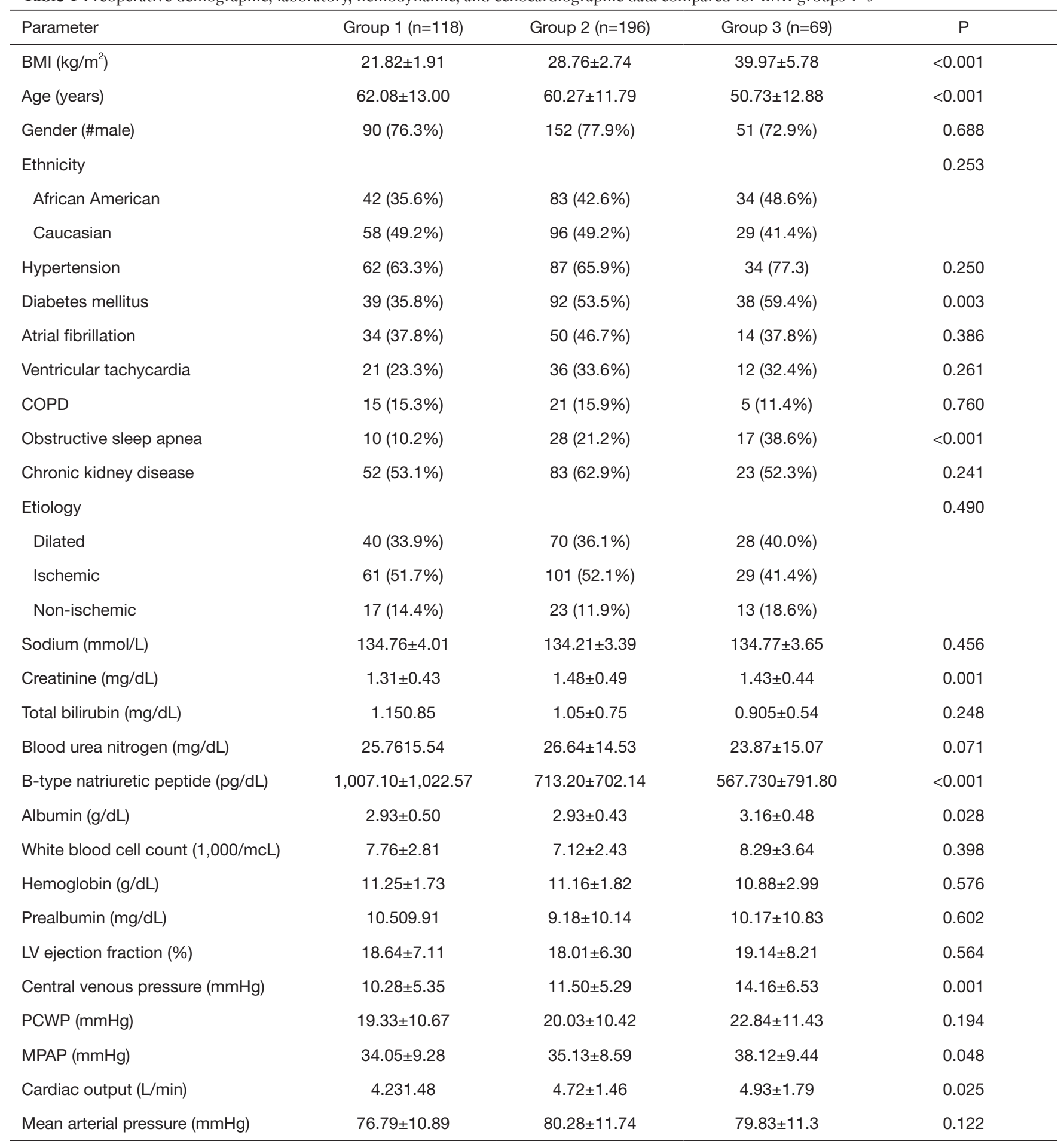

BMI, body mass index; COPD, chronic obstructive pulmonary disease; LV, left ventricular; PCWP, pulmonary capillary wedge pressure; MPAP, mean pulmonary artery pressure. 
Table 2 Survival comparison for BMI groups

\begin{tabular}{lcccc}
\hline Parameter & Group 1 $(\mathrm{n}=118)$ & Group 2 $(\mathrm{n}=196)$ & Group 3 $(\mathrm{n}=69)$ & $\mathrm{P}$ \\
\hline 30-day survival & $115(97.5)$ & $191(97.4)$ & $66(95.7)$ & 0.720 \\
365-day survival & $96(81.4)$ & $171(87.2)$ & $60(87.0)$ & 0.452 \\
730-day survival & $88(74.6)$ & $159(81.1)$ & $57(82.6)$ & 0.623 \\
1,095-day survival & $86(72.9)$ & $145(74.0)$ & $49(71.0)$ & 0.890 \\
\hline
\end{tabular}

Data are shown as $\mathrm{n}(\%)$. BMI, body mass index.

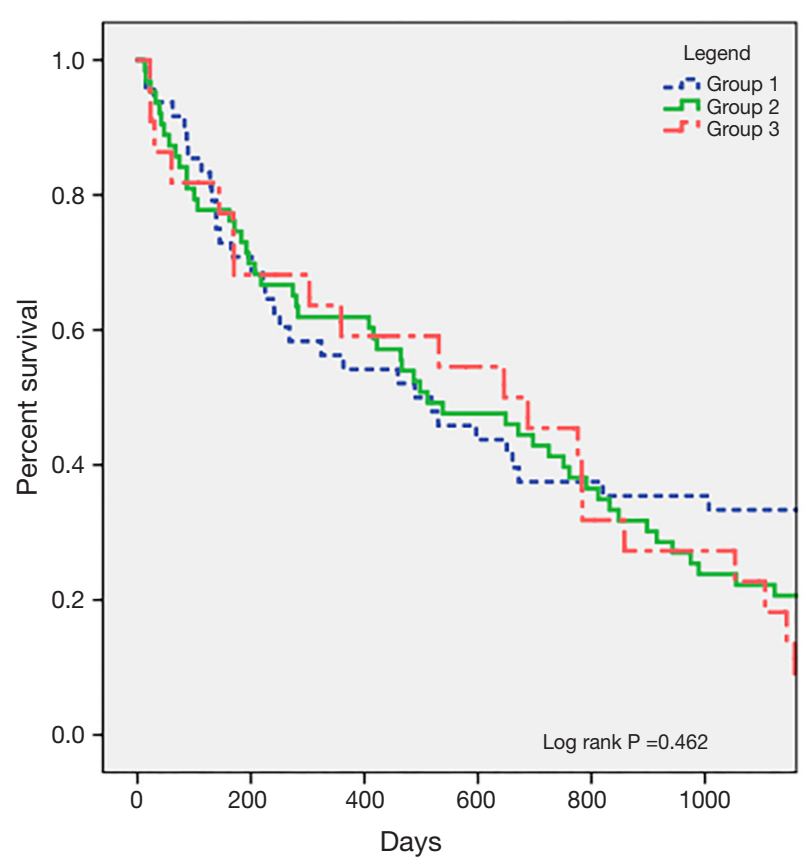

Figure 1 Kaplan-Meier survival curve for the 3 BMI groups. Group 1: underweight and healthy (BMI $<25 \mathrm{~kg} / \mathrm{m}^{2}$ ); group 2: preobesity and obesity $\left(25 \mathrm{~kg} / \mathrm{m}^{2} \leq \mathrm{BMI}<35 \mathrm{~kg} / \mathrm{m}^{2}\right)$, and group 3: extreme obesity (BMI $\geq 35 \mathrm{~kg} / \mathrm{m}^{2}$ ). BMI, body mass index.

in survival between the BMI categories at any time points analyzed (Table 2). Kaplan-Meier survival analysis indicated similar survival functions for the 3 groups (log-rank $\mathrm{P}=0.462$ ) (Figure 1). Cox regression modeling was used to assess survival, with adjustment for baseline characteristics that differed significantly between the BMI categories. This multivariate model indicated no relationship between BMI and postoperative survival (HR: 1.01, CI: 0.930-1.098, $\mathrm{P}=0.810$ ).

\section{Adverse events}

Adverse events, compared between the 3 groups, are shown in Table 3. Patients in group 3 had significantly higher rates of respiratory failure $(\mathrm{P}=0.021)$, and $\mathrm{RV}$ failure $(\mathrm{P}=0.049)$, but lower rates of re-operative bleeding $(\mathrm{P}=0.047)$. There were trends, which did not reach statistical significance, towards higher rates of pump thrombosis and driveline infection in group 3. There were no other differences in adverse events or postoperative length of stay between the groups.

\section{Discussion}

This study investigated outcomes in patients with extreme obesity undergoing LVAD implantation. While LVADs are an effective long-term intervention for end-stage heart failure, expanding rates of obesity and extreme obesity increasingly complicate patient selection for this advanced surgical therapy $(5,6,11)$. Though previous data and guidelines have suggested that LVAD implantation in patients with very low or very high BMI may confer excessive risk, several recent reports indicate no increased mortality in obese patients implanted with LVADs (5,7-12). This study evaluated outcomes in a group of patients with extreme obesity, including 25 patients with BMI greater than $40 \mathrm{~kg} / \mathrm{m}^{2}$ and 4 with BMI greater than $50 \mathrm{~kg} / \mathrm{m}^{2}$. Despite evidence of poor outcomes in obese patients after heart transplantation, our study found no decrement in survival in patients with extreme obesity. This finding supports those of similar studies, and may be related to the "obesity paradox", the hypothesis that obesity is protective against protein calorie malnutrition or cachectic states in heart failure $(13,14)$. This theory is supported by the finding in our study that albumin levels, a common surrogate marker for nutritional status, were significantly higher in the extreme obesity group compared to all others. Recent evidence, however, has indicated that patients with morbid obesity, defined as BMI $>40 \mathrm{~kg} / \mathrm{m}^{2}$, may be excluded from the benefits of the obesity paradox (15). In the 
Table 3 Comparison of adverse events for BMI groups

\begin{tabular}{|c|c|c|c|c|}
\hline Parameter & Group 1 (n=118), n (\%) & Group 2 (n=196), n (\%) & Group 3 (n=69), n (\%) & $\mathrm{P}$ \\
\hline Respiratory failure & $16(13.6)$ & $31(15.8)$ & $20(29.0)$ & 0.021 \\
\hline RV failure & $36(30.5)$ & $74(37.8)$ & $34(49.3)$ & 0.049 \\
\hline Pump thrombosis & $6(5.1)$ & $12(6.1)$ & $7(10.1)$ & 0.205 \\
\hline Driveline/pocket infection & $22(19.5)$ & $39(20.3)$ & $19(27.5)$ & 0.381 \\
\hline Systemic infection & $21(18.6)$ & $42(22.2)$ & $11(16.4)$ & 0.533 \\
\hline Neurologic event & $14(12.4)$ & $17(8.9)$ & $9(13.0)$ & 0.502 \\
\hline Gl bleed & $39(34.5)$ & $65(34.4)$ & $17(25.8)$ & 0.397 \\
\hline
\end{tabular}

$\mathrm{RV}$, right ventricular; Gl, gastrointestinal.

general population, a U-shaped relationship between BMI and mortality has been observed, in which patients with very high and very low BMI fare poorly; as such, further investigation is required to determine whether this trend applies to patients with heart failure and LVAD support (16).

Despite similar post-LVAD survival for all BMI groups, our study indicates that rates of postoperative adverse events, including respiratory failure and RV failure, are higher among patients with extreme obesity. These findings may be attributed to biological problems resulting from obesity. Respiratory compromise in obesity is thought to result from complicated intubation, increased abdominal pressures, and obesity hypoventilation syndrome and may manifest as postoperative respirator dependence or respiratory failure (17). In this study, patients with extreme obesity had significantly higher rates of OSA, suggesting that anatomical airway obstruction may play a role in their respiratory failure. Chronic obesity can also lead to structural changes in the myocardium, increased RV afterload, conduction system abnormalities, inflammation, and direct cardiac lipotoxicity (18). Ventilation-perfusion mismatch due to respiratory compromise can lead to hypoxia induced pulmonary hypertension and subsequent RV failure. Despite higher rates of these two adverse events in group 3, these patients did not have longer postoperative length of stay.

Previous studies have identified increased rates of driveline infection and device thrombosis in obese patients, potentially resulting from impaired immune surveillance and altered hematologic profiles $(8,19)$. Our study notes higher rates of both infection and thrombosis in group 3 which did not reach statistical significance. This represents the need for further research to investigate this relationship and the potential risk for poor outcomes in LVAD patients. This study did find significantly higher rates of re-operative bleeding in group 1 compared to groups 2 and 3 . This trend has been noted in previous research, and is thought to be related to poor production of coagulation and clotting factors in individuals with low BMI due to malnutrition or cachexia.

This study demonstrates no decrease in survival up to 3 years following LVAD implantation in patients with extreme obesity compared to obese, normal, and healthy weight individuals. There were several limitations. First this is a retrospective, single center study and, as a result, may be subject to selection bias. The number of patients in the extreme obesity group, while larger than in previous studies, remained relatively small. Additional study may be valuable to understand postoperative outcomes in these patients.

\section{Conclusions}

The data presented in this study indicated no decrement in survival for patients with $\mathrm{BMI} \geq 35 \mathrm{~kg} / \mathrm{m}^{2}$ undergoing continuous flow LVAD implantation. These patients experience higher rates of postoperative RV failure and respiratory failure, but, with appropriate nutritional optimization, should be considered surgical candidates.

\section{Acknowledgements}

None. 


\section{Footnote}

Conflicts of Interest: The authors have no conflicts of interest to declare.

Ethical Statement: This study was approved by the institutional review board under the protocol \#6250.

\section{References}

1. Ng M, Fleming T, Robinson M, et al. Global, regional, and national prevalence of overweight and obesity in children and adults during 1980-2013: a systematic analysis for the Global Burden of Disease Study 2013. Lancet 2014;384:766-81.

2. Dietz WH, Robinson TN. Clinical practice. Overweight children and adolescents. N Engl J Med 2005;352:2100-9.

3. Wang YC, McPherson K, Marsh T, et al. Health and economic burden of the projected obesity trends in the USA and the UK. Lancet 2011;378:815-25.

4. Kenchaiah S, Evans JC, Levy D, et al. Obesity and the risk of heart failure. N Engl J Med 2002;347:305-13.

5. Russo MJ, Hong KN, Davies RR, et al. The effect of body mass index on survival following heart transplantation: do outcomes support consensus guidelines? Ann Surg 2010;251:144-52.

6. Grady KL, White-Williams C, Naftel D, et al. Are preoperative obesity and cachexia risk factors for post heart transplant morbidity and mortality: a multi-institutional study of preoperative weight-height indices. Cardiac Transplant Research Database (CTRD) Group. J Heart Lung Transplant 1999;18:750-63.

7. Han JJ, Sooppan R, Johnson AP, et al. Higher body mass index increases risk of HeartMate II pump thrombosis but does not adversely affect long term survival. Circ J 2017;81:213-9.

8. Clerkin KJ, Naka Y, Mancini DM, et al. The impact

Cite this article as: Yost G, Coyle L, Gallagher C, Graney N, Siemeck R, Tatooles A, Pappas P, Bhay G. The impact of extreme obesity on outcomes after left ventricular assist device implantation. J Thorac Dis 2017;9(11):4441-4446. doi: $10.21037 /$ jtd.2017.10.52 of obesity on patients bridged to transplantation with continuous-flow left ventricular assist devices. JACC Heart Fail 2016;4:761-8.

9. Mohamedali B, Yost G, Bhat G. Obesity as a risk factor for consideration for left ventricular assist devices. J Card Fail 2015;21:800-5.

10. Brewer RJ, Lanfear DE, Sai-Sudhakar CB, et al. Extremes of body mass index do not impact mid-term survival after continuous-flow left ventricular assist device implantation. J Heart Lung Transplant 2012;31:167-72.

11. Stevenson LW. Selection and management of candidates for heart transplantation. Curr Opin Cardiol 1996;11:166-73.

12. Musci M, Loforte A, Potapov EV, et al. Body mass index and outcome after ventricular assist device placement. Ann Thorac Surg 2008;86:1236-42.

13. Butler J, Howser R, Portner PM, et al. Body mass index and outcomes after left ventricular assist device placement. Ann Thorac Surg 2005;79:66-73.

14. Oga EA, Eseyin OR. The obesity paradox in heart failure: a systematic review of a decade of evidence. J Obes 2016;2016:9040248

15. Nagarajan V, Cauthen CA, Starling RC, et al. Prognosis of morbid obesity patients with advanced heart failure. Congest Heart Fail 2013;19:160-4.

16. Adams KF, Shatzkin A, Harris TB, et al. Overweight, obesity, and mortality in a large prospective cohort of persons 50 to 71 years old. N Engl J Med 2006;355:763-78.

17. Miehsler W. Mortality, morbidity and special issues of obese ICU patients. Wien Med Wochenschr 2010;160:124-8.

18. Ebong IA, Goff DC Jr, Rodriguez CJ, et al. Mechanisms of heart failure in obesity. Obes Res Clin Pract 2014;8:e540-8.

19. Campello E, Zabeo E, Radu CM, et al. Hypercoagulability in overweight and obese subjects who are asymptomatic for thrombotic events. Thromb Haemost 2015;113:85-96. 